\title{
Combined Extension and Torsion of a Swollen Cylinder Within the Context of Mixture Theory
}

\author{
By \\ M. V. Gandhi and M. Usman, East Lansing, Michigan, \\ A. S. Wineman, Ann Arbor, Michigan, \\ and K. R. Rajagopal, Pittsburgh, Pennsylvania
}

With 6 Figures

(Received July 5, 1988; revised October 14, 1988)

\begin{abstract}
Summary
The problem of a cylindrical mixture of a nonlinearly elastic solid and an ideal fluid subjected to combined finite axial extension and torsion is considered. In previous work, a 'universal relation' has been presented by assuming a small angle of twist. In this work, the general problem for the finite deformation of the swollen cylinder is discussed in the context of Mixture Theory. Computational results for the variation of the radial and tangential stretch ratios and the distribution of the fluid in the swollen deformed state are presented. The results demonstrate that the swollen volume of a eylinder reduces with twisting when the axial stretch ratio is held constant. Computational results for the reduction in the swollen volume predict the same qualitative and quantitative trends as observed in experimental results.
\end{abstract}

\section{Introduction}

The combined finite extension and torsion of a cylindrical mixture of a nonlinearly elastic solid and an ideal fluid is considered. In previous work [1], a 'universal relation' has been presented for the case of small twist. In this work, the general problem for the finite deformation of the cylindrical mixture is formulated in the context of Mixture Theory [2], [3] in order to account for the interaction between the solid and fluid constituents. Boundary-value problems for solidfluid mixtures of this kind have been studied previously [1], [4]-[8] in the context of Mixture Theory. The boundary-value problem presented here-in is intrinsically interesting since it is one of the few problems involving large nonhomogeneous deformations for which experimental results are available. Therefore, this boundary-value problem serves as a test-bed for evaluating the validity and predictive capability of Mixture Theory in modeling the interaction of elastic solids and ideal fluids undergoing large deformations. 
The first treatment of the problem of combined finite extension and torsion of a swollen rubber cylinder appears to be due to Treloar [9], where the cylinder is assumed to be saturated with the fluid, and the saturated solid-fluid mixture is treated as an equivalent homogenized continuum. However, in the approach presented here-in the interaction of the solid and the fluid is treated by considering the heterogeneous mixture within the context of Mixture Theory. This formulation permits the analysis of the individual motion of the solid constituent and the fluid constituent by incorporating the interaction between the two. It may also be pointed out that Treloar's approach of treating the saturated solid-fluid mixture as an equivalent homogenized continuum is restricted to equilibrium problems only. However, the formulation within the context of Mixture Theory would permit the investigation of problems where the state of material elements in the domain could range from being completely dry to fully saturated as in the study of time-dependent diffusion problems, for example.

In the problem considered here-in, both the solid and fluid constituents are at rest. However, the fluid can be nonhomogeneously dispersed throughout the mixture domain, which gives rise to gradients in the fluid density. The physical mechanism for the existence of such gradients is provided by the presence of an interaction body force which each constituent exerts on the other.

A brief review of the notations and basic equations relevant to a mixture of interacting continua is presented in Section 2 . The constitutive equations for the mixture of a nonlinearly eastic solid and an ideal fluid are discussed in Section 3. The general problem of combined finite extension and torsion is formulated and discussed within the context of Mixture Theory in Section 4. Computational results for the spatial variation of the radial and tangential stretch ratios and the distribution of the fluid in the swollen deformed state are also presented.

\section{Preliminaries: Notations and Basic Equations}

A brief review of the notations and basic equations of Mixture Theory is presented in this section for completeness and continuity. The historical development and a detailed exposition of the theory are succinctly presented in the comprehensive review articles by Atkin and Craine [2] and Bowen [3].

Let $\Omega$ and $\Omega_{t}$ denote the reference configuration and the configuration of the body at time $t$, respectively. For a function defined on $\Omega \times \boldsymbol{R}$ and $\Omega_{t} \times \boldsymbol{R}, \nabla$ and grad are used to represent the partial derivative with respect to $\Omega$ and $\Omega_{t}$, respectively. Also $\partial / \partial t$ denotes the partial derivative with respect to $t$. The divergence operator related to grad is denoted by div.

The solid-fluid aggregate will be considered a mixture with $S_{1}$ representing the solid and $S_{2}$ representing the fluid. At any instant of time $t$, it is assumed that each place in the space is occupied by particles belonging to both $S_{1}$ and $S_{2}$. Let $\boldsymbol{X}^{(\mathbf{1})}$ and $\boldsymbol{X}^{(2)}$ denote the reference positions of typical particles of $S_{1}$ and $S_{2}$. The 
motion of the solid and the fluid is represented by

$$
x^{(1)}=x_{1}\left(\boldsymbol{X}^{(1)}, t\right), \quad \text { and } \quad x^{(2)}=x_{2}\left(\boldsymbol{X}^{(2)}, t\right)
$$

These motions are assumed to be one-to-one, continuous and invertible. The various kinematical quantities associated with the solid $S_{1}$ and the fluid $S_{2}$ are:

velocity: $\quad \boldsymbol{v}^{(1)}=\frac{D^{(1)} x_{1}}{D t}, \quad \boldsymbol{v}^{(2)}=\frac{D^{(2)} x_{2}}{D t}$,

acceleration: $\quad \boldsymbol{a}^{(1)}=\frac{D^{(1)} \boldsymbol{v}^{(1)}}{D t}, \quad \boldsymbol{a}^{(2)}=\frac{D^{(2)} \boldsymbol{v}^{(2)}}{D t}$

velocity gradient: $\quad \boldsymbol{L}^{(1)}=\frac{\partial \boldsymbol{v}^{(1)}}{\partial \boldsymbol{x}^{(1)}}, \quad \boldsymbol{L}^{(2)}=\frac{\partial \boldsymbol{v}^{(2)}}{\partial \boldsymbol{x}^{(2)}}$,

rate of deformation tensor:

$$
\boldsymbol{D}^{(1)}=\frac{1}{2}\left(\boldsymbol{L}^{(1)}+\boldsymbol{L}^{(1) \mathrm{T}}\right), \quad \boldsymbol{D}^{(2)}=\frac{1}{2}\left(\boldsymbol{L}^{(2)}+\boldsymbol{L}^{(2) \mathrm{T}}\right),
$$

where $D^{(1)} / D t$ denotes differentiation with respect to $t$, holding $\boldsymbol{x}^{(\mathbf{1})}$ fixed, and $D^{(2)} / D t$ denotes a similar operation holding $x^{(2)}$ fixed. The deformation gradient $\boldsymbol{F}$ associated with the solid is given by

$$
\boldsymbol{F}=\frac{\partial \boldsymbol{x}_{1}}{\partial \boldsymbol{X}^{(1)}}
$$

The total density of the mixture $\varrho$ and the mean velocity of the mixture $v$ are defined by

and

$$
\varrho=\varrho_{1}+\varrho_{2}
$$

$$
\varrho v=\varrho_{1} \boldsymbol{v}^{(1)}+\varrho_{2} \boldsymbol{v}^{(2)},
$$

where $\varrho_{1}$ and $\varrho_{2}$ are the densities of the solid and the fluid in the mixed state, respectively, defined per unit volume of the mixture at time $t$.

The basic equations of Mixture Theory are presented next.

(i) Conservation of Mass

Assuming no interconversion of mass between the two interacting continua, the appropriate forms for the conservation of mass for the solid and the fluid are

and

$$
\varrho_{1}|\operatorname{det} F|=\varrho_{10},
$$

$$
\frac{\partial \varrho_{2}}{\partial t}+\operatorname{div}\left(\varrho_{2} \boldsymbol{v}^{(2)}\right)=0
$$

where $\varrho_{10}$ is the mass density of the solid in the reference state. 


\section{(ii) Conservation of Linear Momentum}

Let $\sigma$ and $\boldsymbol{\pi}$ denote the partial stress tensors associated with the solid $S_{1}$ and the fluid $S_{2}$, respectively. Then, assuming that there are no external body forces, the balance of linear momentum for the solid and fluid are given by

$$
\operatorname{div} \boldsymbol{\sigma}-\boldsymbol{b}=\varrho_{1} \boldsymbol{a}^{(1)},
$$

and

$$
\operatorname{div} \boldsymbol{\pi}+\boldsymbol{b}=\varrho_{2} \boldsymbol{a}^{(2)} .
$$

In Eqs. (2.11) and (2.12), $b$ denotes the interaction body force vector, which accounts for the mechanical interaction between the solid and the fluid.

(iii) Conservation of Angular Momentum

This condition states that

$$
\sigma+\boldsymbol{\pi}=\sigma^{\top}+\boldsymbol{\pi}^{\top}
$$

However, the partial stresses $\sigma$ and $\pi$ need not be symmetric.

(iv) Surface Conditions

Let $\boldsymbol{t}$ and $\boldsymbol{q}$ denote the surface traction vectors taken by $S_{1}$ and $S_{2}$, respectively, and let $\boldsymbol{n}$ denote the unit outer normal vector at a point on the surface of the mixture domain. Then the partial surface tractions are related to the partial stress tensors by

$$
\boldsymbol{t}=\boldsymbol{\sigma}^{\top} \boldsymbol{n}, \text { and } \boldsymbol{q}=\boldsymbol{\pi}^{\top} \boldsymbol{n}
$$

\section{(v) Thermodynamical Considerations}

The laws of conservation of energy and the entropy production inequality are not explicitly mentioned here for brevity. However, the relevant results are quoted. A complete discussion of these issues is presented in [10].

Let the Helmholtz free energy per unit mass of $S_{1}$ and $S_{2}$ be denoted by $A_{1}$ and $A_{1}$, respectively. The Helmholtz free energy per unit mass of the mixture is defined by

$$
\varrho A=\varrho_{1} A_{1}+\varrho_{2} A_{2}
$$

Note that by setting

$$
\begin{aligned}
& \boldsymbol{b}=-\operatorname{grad} \phi_{1}+\overline{\boldsymbol{b}}=-\operatorname{grad} \phi_{2}+\overline{\boldsymbol{b}}, \\
& \sigma=\phi_{1} \boldsymbol{I}+\overline{\boldsymbol{\sigma}}, \\
& \boldsymbol{x}=\phi_{2} \boldsymbol{I}+\overline{\boldsymbol{x}}
\end{aligned}
$$

where,

$$
\phi_{1}=\varrho_{1}\left(A_{1}-A\right), \quad \phi_{2}=\varrho_{2}\left(A_{2}-A\right), \quad \phi_{1}+\phi_{2}=0,
$$


Eqs. (2.11)-(2.13) become

$$
\begin{aligned}
& \operatorname{div} \overline{\bar{\sigma}}-\bar{b}=\varrho_{1} a^{(1)}, \\
& \operatorname{div} \overline{\boldsymbol{x}}+\overline{\boldsymbol{b}}=\varrho_{2} \boldsymbol{a}^{(2)}, \\
& \overline{\boldsymbol{\sigma}}+\overline{\boldsymbol{x}}=\overline{\boldsymbol{\sigma}}^{\mathrm{T}}+\overline{\boldsymbol{x}}^{\mathrm{T}} .
\end{aligned}
$$

The terms in Eqs. (2.16)-(2.18) which depend on $\phi_{1}$ and $\phi_{2}$ do not contribute to the equations of motion or the total stress tensor.

(vi) Volume Additivity Assumption

Attention is restricted to a mixture of incompressible materials. It is assumed that the volume of the mixture at any given time is the sum of the volumes occupied by the solid and fluid constituents at that time. This implies that the motion of the interacting continua is such that it satisfies the following relationship [11\},

$$
\frac{\varrho_{1}}{\varrho_{10}}+\frac{\varrho_{2}}{\varrho_{20}}=1
$$

where $\varrho_{20}$ is the mass density of the fluid in the reference state:

\section{Constitutive Equations}

A mixture of an elastic solid and a fluid is considered. The solid is assumed to be nonlinearly elastic, and the fluid is assumed to be ideal. Thus all the constitutive functions are required to depend on the following variables:

$$
\boldsymbol{F}, \nabla \boldsymbol{F}, \varrho_{2}, \operatorname{grad} \varrho_{2}, T, \operatorname{grad} T, \boldsymbol{v}^{(1)} \text { and } \boldsymbol{v}^{(2)},
$$

where $T$ denotes the common absolute temperature of the solid and the fluid.

A lengthy but standard argument, based on the balance of energy, entropy production inequality, restrictions due to material frame indifference and the assumption that the solid is isotropic in its reference state, leads to the following results [4].

The constitutive equations are written in terms of the Helmholtz free energy function $A$ per unit mass of the mixture, and the form of this function is given by

$$
A=\hat{A}\left(I_{1}, I_{2}, I_{3}, \varrho_{2}, T\right),
$$

where $I_{1}, I_{2}, I_{3}$ are the principal invariants of $\boldsymbol{B}=\boldsymbol{F} \boldsymbol{F}^{\top}$ defined through

$$
\begin{aligned}
& I_{1}=\operatorname{tr} B, \\
& I_{2}=\frac{1}{2}\left[(\operatorname{tr} B)^{2}-\operatorname{tr} B^{2}\right],
\end{aligned}
$$

and

$$
I_{3}=\operatorname{det} \boldsymbol{B}=(\operatorname{det} \boldsymbol{F})^{2} .
$$


Using (2.9), (2.22) and (3.4), $I_{3}$ can be expressed in terms of $\varrho_{2}$ by the relation

$$
I_{3}{ }^{1 / 2}=\operatorname{det} \boldsymbol{F}=\left(1-\varrho_{2} / \varrho_{20}\right)^{-1} .
$$

Furthermore, on restricting attention to isothermal conditions Eq. (3.1) reduces to

$$
A=A\left(I_{1}, I_{2}, \varrho_{2}\right)
$$

The components of the partial stress tensor for the solid and fluid, and the interaction body force vector for isothermal conditions are given by

$$
\begin{aligned}
& \bar{\sigma}_{k i}=-p \frac{\varrho_{1}}{\varrho_{10}} \delta_{k i}+2 \varrho\left\{\left(\frac{\partial A}{\partial I_{1}}+\frac{\partial A}{\partial I_{2}} I_{1}\right) B_{k i}-\frac{\partial A}{\partial I_{2}} B_{k m} B_{m i}\right\}, \\
& \bar{\pi}_{k i}=-p \frac{\varrho_{2}}{\varrho_{20}} \delta_{k i}-\varrho \varrho_{2} \frac{\partial A}{\partial \varrho_{2}} \delta_{k i},
\end{aligned}
$$

and

$$
\begin{aligned}
\bar{b}_{k}= & -\frac{p}{\varrho_{10}} \frac{\partial \varrho_{1}}{\partial x_{k}}+\varrho_{1} \frac{\partial A}{\partial \varrho_{2}} \frac{\partial \varrho_{2}}{\partial x_{k}}-\varrho_{2}\left\{\left(\frac{\partial A}{\partial I_{1}}+\frac{\partial A}{\partial I_{2}} I_{1}\right) \delta_{i l}-\frac{\partial A}{\partial I_{2}} B_{i l}\right\} \\
& \cdot B_{i l, k}+\alpha \frac{\varrho_{1}}{\varrho_{10}} \frac{\varrho_{2}}{\varrho_{20}}\left(v_{k}^{(1)}-v_{k}^{(2)}\right) .
\end{aligned}
$$

In Eqs. (3.7)-(3.9), $p$ is a scalar which arises due to the incompressibility constraint. The constitutive parameter $\alpha$ accounts for a contribution to the interaction body force due to relative motion between the solid and the fluid. The interaction between the solid and the fluid is evident in these equations, where the partial stress of each constituent is affected by the deformed state of both the constituents. It is also useful to record the representation for the components of the total stress tensor

$$
\begin{aligned}
T_{k i}= & \bar{\sigma}_{k i}+\bar{\pi}_{k i}=-p \delta_{k i}-\varrho \varrho_{2} \frac{\partial A}{\partial \varrho_{2}} \delta_{k i}+2 \varrho \\
& \cdot\left\{\left(\frac{\partial A}{\partial I_{1}}+\frac{\partial A}{\partial I_{2}} I_{1}\right) B_{k i}-\frac{\partial A}{\partial I_{2}} B_{k m} B_{m i}\right\}
\end{aligned}
$$

In the remainder of this paper, only $\overline{\boldsymbol{\sigma}}$, and $\overline{\boldsymbol{\pi}}$ and $\overline{\boldsymbol{b}}$, will be used. Hence, for notational convenience, the superposed bars are dropped.

\section{Combined Extension and Torsion of a Swollen Cylinder}

Consider a solid circular cylinder described by a radius $R_{0}$ and a length $L_{0}$ in the reference configuration. The co-ordinates of a material particle in the reference configuration will be denoted by cylindrical coordinates $(R, \Theta, Z)$. The cylinder 
is assumed to be subjected to the following deformation:

$$
r=r(R), \quad \theta=\Theta+\psi \lambda Z, \quad \text { and } \quad z=\lambda Z,
$$

where $(r, \theta, z)$ denote the co-ordinates of the particle at $(R, \Theta, Z)$ in the deformed swollen configuration, $\lambda$ and $\psi$ being constants. The above deformation corresponds to a finite elongation (with an associated stretch ratio $\lambda$ ) along the $z$-coordinate direction, followed by a rotation of $\psi$ per unit current length.

The Cauchy-Green tensor $\boldsymbol{B}$ which is defined as

$$
\boldsymbol{B}=\boldsymbol{F} \boldsymbol{F}^{\top}
$$

takes the following form for the above deformation:

$$
\begin{aligned}
\boldsymbol{B} & =\left(\begin{array}{lll}
\left(\frac{d r}{d R}\right)^{2} & 0 & 0 \\
0 & \left(\frac{r}{R}\right)^{2}+(\psi \lambda r)^{2} & \psi \lambda^{2} r \\
0 & \psi \lambda^{2} r & \lambda^{2}
\end{array}\right), \\
& =\left(\begin{array}{llll}
\lambda_{r}^{2} & 0 & 0 \\
0 & \lambda_{\theta}^{2}+\left(\psi R \lambda \lambda_{\theta}\right)^{2} & \psi \lambda^{2} \lambda_{\theta} R \\
0 & \psi \lambda^{2} \lambda_{\theta} R & \lambda^{2}
\end{array}\right),
\end{aligned}
$$

where $\lambda_{r}=d r / d R$ and $\lambda_{\theta}=r / R$ denote the stretch ratios in the $r$ and $\theta$ directions, respectively. The principal invariants of $\boldsymbol{B}$ are then given as

$$
\begin{aligned}
& I_{1}=\lambda_{r}^{2}+\lambda_{\theta}^{2}\left(1+\psi^{2} R^{2} \lambda^{2}\right)+\lambda^{2}, \\
& I_{2}=\lambda^{2}\left(\lambda_{r}{ }^{2}+\lambda_{\theta}{ }^{2}\right)+\lambda_{\theta}{ }^{2} \lambda_{r}^{2}\left(1+\psi^{2} R^{2} \lambda^{2}\right),
\end{aligned}
$$

and

$$
I_{3}=\lambda_{r}^{2} \lambda_{\theta}^{2} \lambda^{2}
$$

The balance of mass equation for the solid constituent (2.9) may be expressed in terms of the stretch ratios as

$$
\frac{\varrho_{1}}{\varrho_{10}}=\frac{1}{\lambda_{r} \lambda_{\theta} \lambda}=v_{1}
$$

where $\nu_{1}$ represents the volume fraction of the solid.

The equations of equilibrium which are appropriate for the deformation being considered are documented next. Since the assumed form of deformation implies that the stresses depend only on the radial co-ordinate $r$, the equations of equilibrium for the solid constituent, namely (2.11), reduce to

$$
\frac{d \sigma_{r r}}{d r}+\frac{\sigma_{r r}-\sigma_{\theta \theta}}{r}-b_{r}=0,
$$


where $\sigma_{r r}$ and $\sigma_{\theta \theta}$ denote the appropriate components of $\sigma$, and $b_{r}$ denotes the component of the interaction body force $b$ in the radial direction. The equilibrium equations for the fluid constituent, namely (2.12), reduce to

$$
\frac{d \pi_{r r}}{d r}+\frac{\pi_{r r}-\pi_{\theta \theta}}{r}+b_{r}=0
$$

where $\pi_{r r}$ and $\pi_{\theta \theta}$ denote the appropriate components of $\boldsymbol{\pi}$. Equations (3.10), (4.9) and (4.10) yield

$$
\frac{d T_{r r}}{d r}+\frac{T_{r r}-T_{\theta \theta}}{r}=0
$$

which is the equation of equilibrium for the mixture.

For the deformation under consideration, it follows from Eq. (4.4) and Eqs. (3.7)-(3.9) that the non-zero components of the partial stress tensors for the solid and fluid constituents are given by

$$
\begin{gathered}
\sigma_{r r}=-p \frac{\varrho_{1}}{\varrho_{10}}+2 \varrho\left(A_{1}+A_{2} I_{1}\right) \lambda_{\mathrm{r}}^{2}-2 \varrho\left(A_{2}\right) \lambda_{\mathrm{r}}^{4}, \\
\dot{\sigma}_{\theta \theta}=-p \frac{\varrho_{1}}{\varrho_{10}}+2 \varrho\left(A_{1}+A_{2} I_{1}\right) \lambda_{\theta}^{2}\left(1+\psi^{2} R^{2} \lambda^{2}\right) \\
-2 \varrho A_{2}\left\{\lambda_{\theta}^{4}\left(1+\psi^{2} R^{2} \lambda^{2}\right)^{2}+\psi^{2} R^{2} \lambda^{4} \lambda_{\theta}^{2}\right\} \\
\sigma_{z z}=-p \frac{\varrho_{1}}{\varrho_{10}}+2 \varrho\left(A_{1}+A_{2} I_{1}\right) \lambda^{2}-2 \varrho A_{2}\left\{\lambda^{4}\left(1+\psi^{2} R^{2} \lambda_{\theta}^{2}\right)\right\}, \\
\sigma_{\theta z}=2 \varrho \psi R\left\{\left(A_{1}+A_{2} I_{1}\right) \lambda^{2} \lambda_{\theta}-A_{2}\left[\lambda^{2} \lambda_{\theta}\left\{\left(1+\psi^{2} R^{2} \lambda^{2}\right) \lambda_{\theta}^{2}+\lambda^{2}\right)\right]\right\},
\end{gathered}
$$

and

$$
\pi_{r r}=\pi_{\theta \theta}=\pi_{z z}=-p \frac{\varrho_{2}}{\varrho_{20}}-\varrho_{2} A_{\varrho_{z}}
$$

respectively.

The only non-zero component of the interaction body force vector is given by

$$
\begin{aligned}
b_{r}= & -\frac{p}{\varrho_{10} \lambda_{r}} \frac{d \varrho_{1}}{d R}+\varrho_{\mathrm{r}} A_{\varrho_{\mathrm{a}}} \frac{d \varrho_{2}}{\lambda_{r} d R} \\
& -\varrho_{2} A_{1} \frac{d}{\lambda_{r} d R}\left\{\lambda_{r}^{2}+\lambda_{\theta}{ }^{2}+\psi^{2} \lambda^{2} R^{2} \lambda_{\theta}{ }^{2}+\lambda^{2}\right\} \\
& -\varrho_{2} A_{2} \frac{d}{\lambda_{r} d R}\left(\lambda_{r}{ }^{2} \lambda^{2}+\lambda_{\theta}{ }^{2} \lambda^{2}+\lambda_{r}{ }^{2} \lambda_{\theta}{ }^{2}\left(1+\psi^{2} R^{2} \lambda^{2}\right)\right),
\end{aligned}
$$

where $A_{1}=\frac{\partial A}{\partial I_{1}}, A_{2}=\frac{\partial A}{\partial I_{2}}$ and $A_{\varrho_{2}}=\frac{\partial A}{\partial \varrho_{2}}$. 
It is sufficient to satisfy any two of the three equilibrium equations (4.9) to (4.11). Equations (4.12)-(4.17) are substituted into the equilibrium equations for the solid and the mixture (4.9) and (4.11), respectively, to get the following functional forms of the equilibrium equations which are stated in terms of the coordinates in the reference configuration for computational convenience:

$$
-\frac{d p}{d R} \frac{\varrho_{1}}{\varrho_{10}}+g_{1}\left(A_{1}, A_{2}, A_{g_{2}}, \lambda_{t}, \lambda_{\theta}, R, \lambda_{\tau}{ }^{\prime}, \lambda_{\theta}{ }^{\prime}, \lambda, \psi^{2} R^{2}\right)=0
$$

and

$$
-\frac{d p}{d R}+g_{2}\left(A_{1}, A_{2}, A_{\varrho_{2}}, \lambda_{r}, \lambda_{\theta}, R, \lambda_{r}^{\prime}, \lambda_{\theta}^{\prime}, \lambda, \psi^{2} R^{2}\right)=0
$$

In Eqs. (4.18) and (4.19) the prime denotes differentiation with respect to the reference radial coordinate $R$, and the radial and tangential stretch ratio $\lambda_{r}$ and $\lambda_{\theta}$, respectively, are related through the compatibility condition given by

$$
\frac{d \lambda_{\theta}}{d R}=\frac{\lambda_{r}-\lambda_{\theta}}{R}
$$

Subsequently, the mixture is assumed to be of a "Neo-Hookean-type", that is, $A$ is a linear function of $I_{1}$, For this case the explicit forms of the equilibrium equations for the mixture and the solid are given by

$$
\begin{aligned}
& -\frac{d p}{d R}-\frac{d}{d R}\left(\varrho \varrho_{2} \frac{\partial A}{\partial \varrho_{2}}\right)+2 \varrho A_{1} \lambda_{r} \\
& \cdot\left[\frac{d \lambda_{r}}{d R}-\frac{\lambda_{r}}{\lambda_{\theta} R}\left(\lambda_{r}-\lambda_{\theta}\right)-\frac{1}{\lambda_{\theta} R}\left(\lambda_{\theta}^{2}-\lambda_{r}^{2}+\psi^{2} \lambda^{2} R^{2} \lambda_{\theta}{ }^{2}\right)\right]=0
\end{aligned}
$$

and

$$
\begin{aligned}
& -\nu_{1} \frac{d p}{d R}+2 \varrho A_{1} \lambda_{r}\left[\frac{d \lambda_{r}}{d R}-\frac{\lambda_{r}}{\lambda_{\theta} R}\left(\lambda_{r}-\lambda_{\theta}\right)-\frac{1}{\lambda_{\theta} R}\left(\lambda_{\theta}^{2}-\lambda_{r}^{2}+\psi^{2} \lambda^{2} R^{2} \lambda_{\theta}^{2}\right)\right] \\
& -\varrho_{1} \varrho_{20} \frac{\partial A}{\partial \varrho_{2}} \nu_{1}\left[\frac{1}{\lambda_{r}} \frac{d \lambda_{r}}{d R}+\frac{1}{\lambda_{\theta}} \frac{\left(\lambda_{r}-\lambda_{\theta}\right)}{R}\right] \\
& +2 \varrho_{2} A_{1}\left[\lambda_{r} \frac{d \lambda_{r}}{d R}+\lambda_{\theta} \frac{\left(\lambda_{r}-\lambda_{\theta}\right)}{R}+\psi^{2} \lambda^{2} \lambda_{\theta} \lambda_{r} R\right]=0 .
\end{aligned}
$$

Equations (4.20)-(4.22) may be solved for $p, \lambda_{r}$ and $\lambda_{\theta}$ once the specific form of the Helmholtz free energy function for the mixture is known, and the appropriate boundary conditions are specified.

The Helmholtz free energy function per unit mass of the mixture is assumed [12] to be given by

$$
A=\frac{\nu_{1}}{\varrho}\left\{\frac{\hat{R} T \varrho_{10}}{2 M_{c}}\left(I_{1}-3\right)+\frac{\hat{R} T}{V_{1}}\left[\frac{1-\nu_{1}}{\nu_{1}} \ln \left(1-\nu_{1}\right)+\chi\left(1-\nu_{1}\right)\right]\right\}
$$


where,

$V_{1}$ is the molar volume of the fluid,

$\chi \quad$ is a constant which depends on the particular combination of the solid and the fluid,

$\hat{R} \quad$ is the universal gas constant,

$T$ is the absolute temperature,

$M_{c}$ is the molecular weight of the polymeric solid between the crosslinks.

Two of the appropriate boundary conditions for solving the set of ordinary differential equations $(4.20)-(4.22)$ are given by

$$
\lambda_{r}(0)=\lambda_{\theta}(0) \text {, }
$$

and

$$
T_{r r}\left(R_{0}\right)=0 .
$$

The boundary condition given by Eq. (4.24) arises due to the compatibility requirement between the radial and tangential stretch ratios at the axis of the cylinder. The boundary condition on the total traction vector represented by Eq. (4.25) is a consequence of the requirement that the outer surface of the cylinder be traction-free. Since a boundary condition for the partial traction vector is not physically obvious, following the arguments presented in [4]- [6] it is assumed that the outer surface of the cylinder is in a saturated state 1 . This assumption results in the boundary condition represented by

$$
S_{r r}\left(R_{0}\right)=0,
$$

where $S_{r r}$ represents the radial stress component for a saturated state, and is given by [5]

$$
S_{r \tau}=\varrho\left(\varrho_{20}-\varrho_{2}\right) \frac{\partial A}{\partial \varrho_{2}}+\varrho_{20} A+2 \varrho A_{1} \lambda_{r}{ }^{2}
$$

The governing equations (4.20)-(4.22) for the combined extension and torsion of a swollen cylinder are highly nonlinear and coupled, and may be solved numerically for the variables $\lambda_{\tau}, \lambda_{\theta}$ and $p$. For computational convenience, Eqs. (4.21) and (4.22) may be combined to eliminate $p$, and for the Helmholtz free energy function given by (4.23) the resulting equation is given by

$$
\frac{R \lambda_{\theta}}{\lambda_{r}} \frac{d \lambda_{r}}{d R}=-\frac{\left(\lambda_{r}-\lambda_{\theta}\right)\left[K\left(2 \chi-\frac{1}{1-\nu_{1}}\right) \nu_{1}-\lambda_{r} \lambda_{\theta}\right]+\psi^{2} \lambda^{2} R^{2} \lambda_{\theta}^{2} \lambda_{r}}{K\left(2 \chi-\frac{1}{1-\nu_{1}}\right) \nu_{1}-\lambda_{r}^{2}}
$$

1 The first two authors have recently shown that this assumption is consistent with Mixture Theory; and results in the entire domain of the cylinder being saturated [14]. 
where,

$$
K=\frac{M_{c}}{\varrho_{10} V_{1}} .
$$

The set of ordinary differential equations given by (4.20) and (4.28) subjected to boundary conditions given by (4.24) and (4.26) were solved numerically. The following material properties [13] were used for the numerical calculations:

Density of rubber in the reference state

Density of solvent in the reference state

$$
\begin{array}{ll}
\varrho_{10}=.9016 & \mathrm{gm} / \mathrm{cc} \\
\varrho_{20}=.862 & \mathrm{gm} / \mathrm{cc} \\
V_{1}=106.0 & \mathrm{cc} / \mathrm{mole}
\end{array}
$$

Molar volume of the solvent

The molecular weight of rubber

between cross links

$M_{\mathrm{c}}=8891.0 \mathrm{gm} / \mathrm{mole}$

Rubber-solvent interaction constant

$\chi=.400$

The numerical value of the universal gas constant $\hat{R}$ is given by $8.317 \times 10^{7}$ dyne-cm $/$ mole $-{ }^{\circ} \mathrm{K}$, and the absolute temperature $T$ was assumed to be $303.16^{\circ} \mathrm{K}$. The computational results are presented in Figs. 1-6 for a value of the axial stretch ratio $\lambda=1.938$ which was maintained in the experimental work presented in [13].

Figure 1 shows the variation of the radial and circumferential stretch ratios for two different values of the angle of twist $\psi$. For the case of no twist $(\psi=0)$ the cylinder is homogeneously swollen whereby the radial and tangential stretch ratios are equal and constant throughout the domain. However, when the swollen cylinder undergoes finite torsion $(\psi=1.0)$ significant gradients in the stretch ratios are evident. Furthermore, even in the case of finite torsion, the deformation in the axial domain is relatively homogeneous, and the gradients in the stretch ratios increase with the radial co-ordinate. Figure 2 shows the variation of the

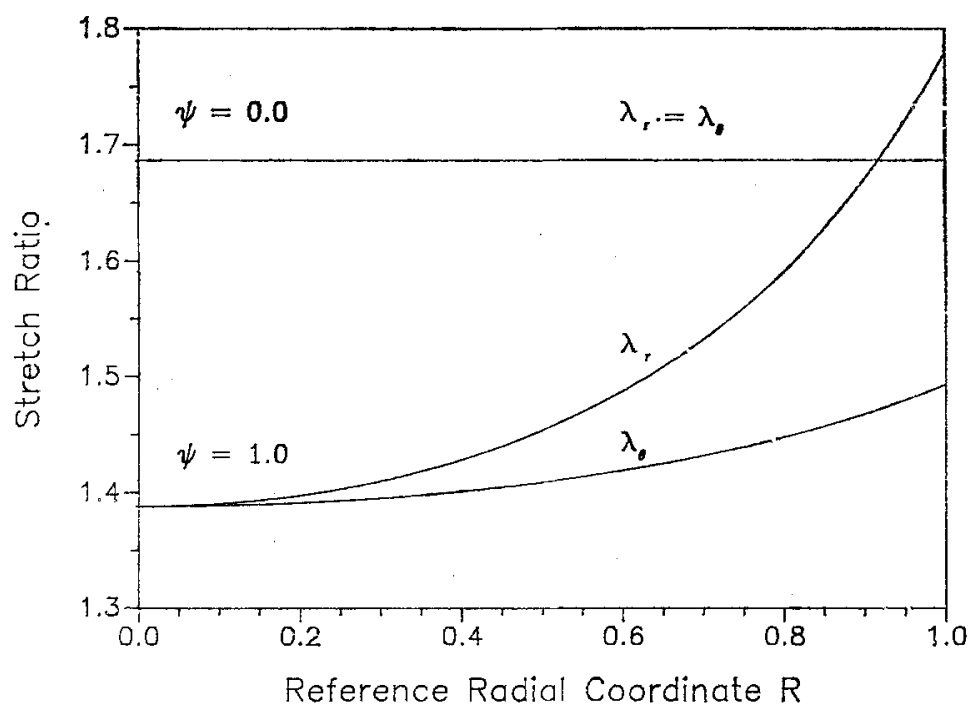

Fig. 1 
M. V. Gandhi, M. Usman, A. S. Wineman, and K. R. Rajagopal:

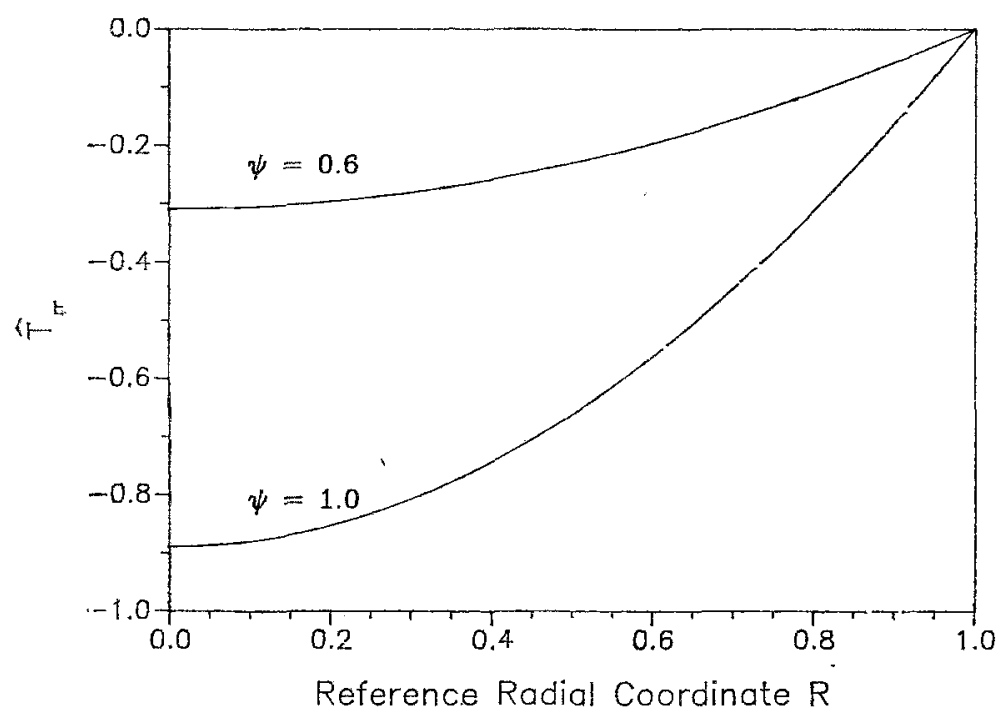

Fig. 2

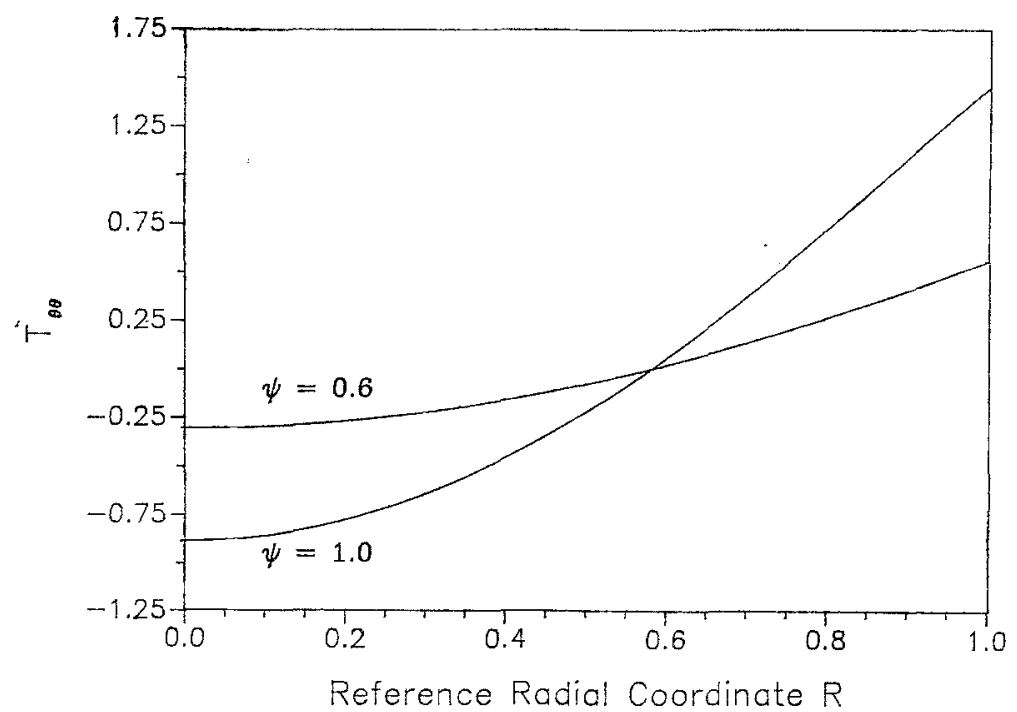

Fig. 3

radial stress for two different values of the angle of twist. It is seen from Fig. 2 that the non-dimensional radial stress is compressive and approaches zero at $R=R_{0}$ due to the boundary condition (4.26) which requires the outer surface of the deformed cylinder to be traction-free. The corresponding variation of the non-dimensional circumferential stress is shown in Fig. 3. The non-dimensional radial and circumferential stresses in Figs. 2 and 3 denoted by $\hat{T}_{r r}$ and $\hat{T}_{\theta \theta}$ have been non-dimensionalized with respect to $\frac{\hat{R}_{\varrho_{10}}}{M_{c}}$. It is clear from Figs. 1, 2, and 3 that the gradients of the radial and circumferential stretch ratios and stresses 


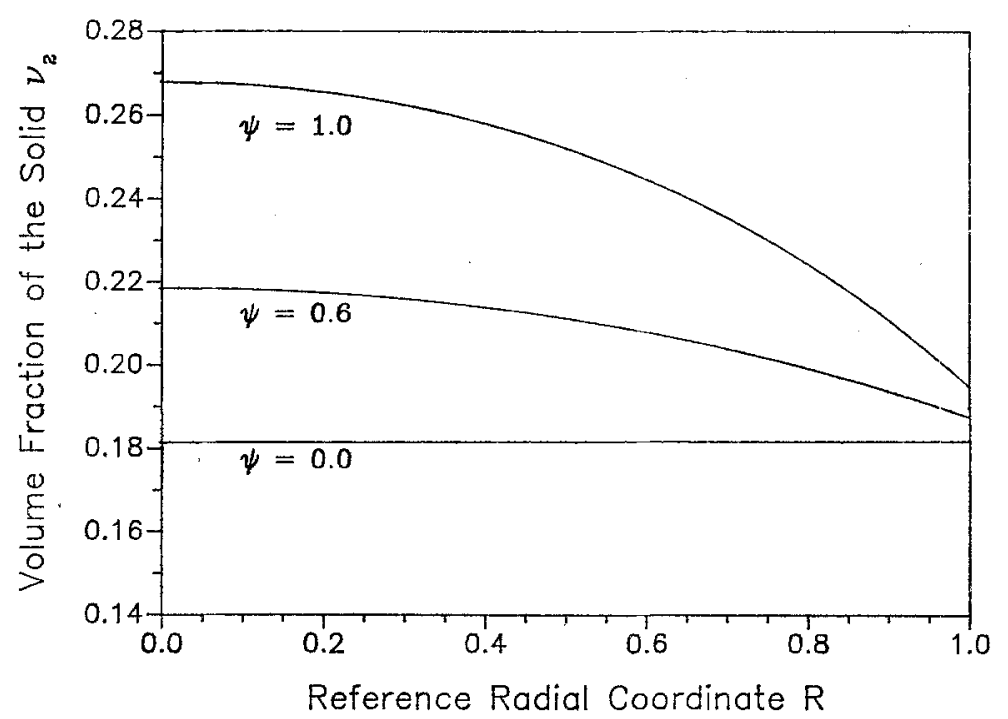

Fig. 4

increase with increasing twist. The variation of the volume fraction of the solid along the reference radial co-ordinate is shown in Fig. 4 for three different values of the angle of twist. It is evident from Fig. 4 that the fluid leaves the swollen deformed cylinder as the angle of twist is increased. The non-dimensional ratio of the current volume $V$ (in the swollen twisted state) to the volume of the original unswollen rubber cylinder $V_{u}$ is presented in Fig. 5. It is clear from these results that as the angle of twist increases the fluid leaves the cylindrical mixture resulting in the reduction of the current volume of the swollen cylinder. Finally, the

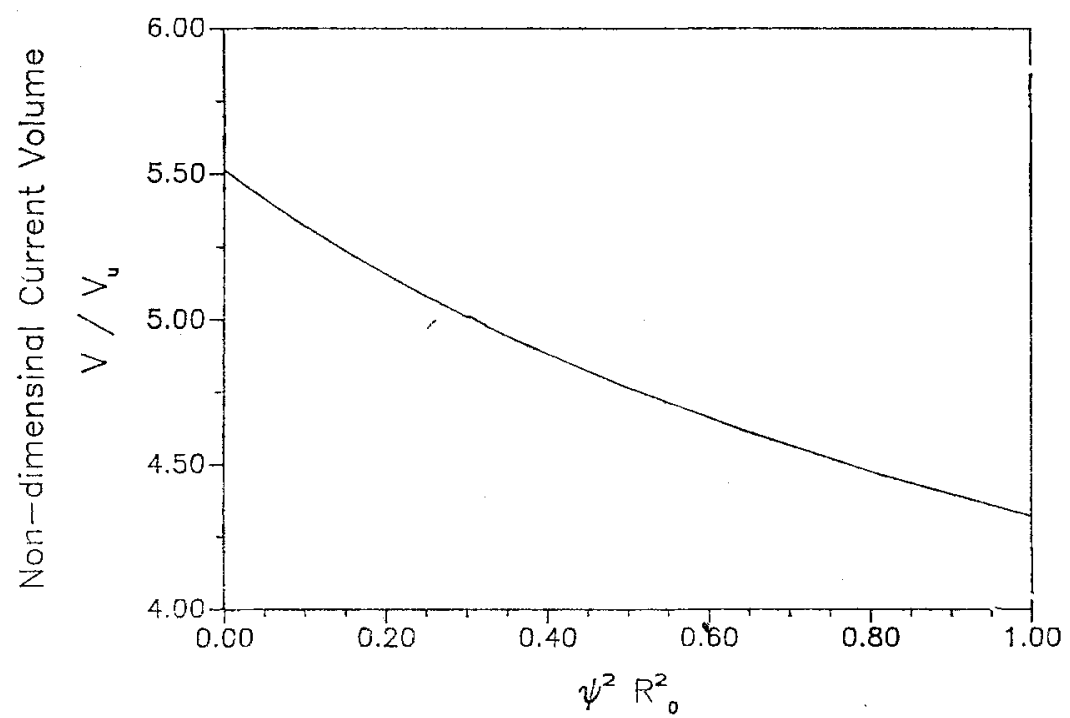

Fig. 5 


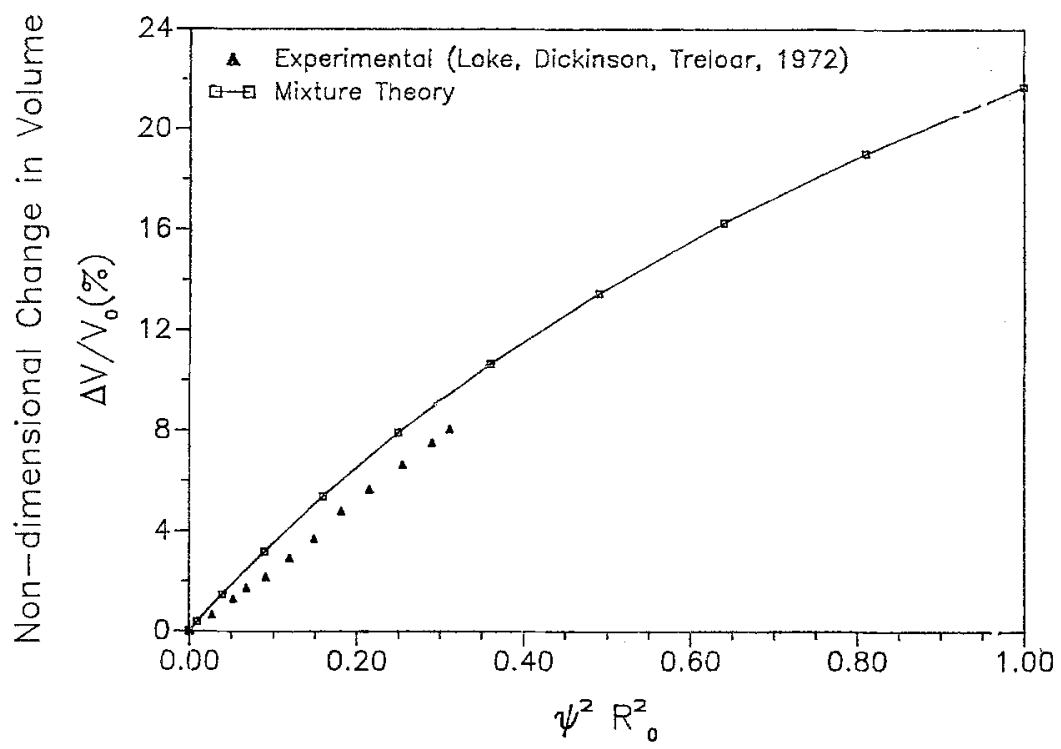

Fig. 6

ratio of the change in the volume $\Delta V=\left(V-V_{0}\right)$ to the saturated swollen untwisted volume $V_{0}$ is compared with experimental results [13] in Fig. 6. The computational results based on mixture theory predict the same qualitative and quantitative trends as observed in experimental results, thereby illustrating the value of employing mixture theory in modeling the interaction of elastic solids and ideal fluids undergoing large deformations.

\section{Acknowledgements}

M. V. Gandhi gratefully acknowledges the support of the National Science Foundation through Grant No. MSM 8514087, the Deferse Advanced Research Projects Agency through Contract No. DAAL 03-87-K-018 and the Michigan Research Excellence and Economic Development Fund.

\section{References}

[1] Gandhi, M. V., Rajagopal, K. R., Wineman, A. S.: A universal relation in torsion for a mixture of solid and fluid. Journal of Elasticity 15 (2), 155-166 (1985).

[2] Atkin, R. J., Craine, R. E. : Continuum theories of mixtures: basic theory and historica development. Q. J. Mech. Appl. Math. 29 (2), 209-244 (1976).

[3] Bowen, R. M.: Continuum physics (Eringen, A. C., ed.), Vol. 3. New York: Academic Press 1975.

[4] Shi, J. J., Wineman, A. S., Rajagopal, K. R.: Applications of the theory of interacting continua to the diffusion of a fluid through a nonlinear elastic medium. Int. J. Engng. Sci. 9, $871-889$ (1981). 
[5] Rajagopal, K. R., Wineman, A. S., Gandhi, M. V.: On boundary conditions for a certain elass of problems in mixture theory. Int. J. Engng. Sci, 24 (8), 1453-1463 (1986).

[6] Gandhi, M. V., Rajagopal, K. R., Wineman, A. S.: Some nonlinear diffusion problems within the context of the theory of interacting continua. Int. J. Engng. Sci. 25 (11/12), $1441-1457$ (1987).

[7] Gandhi, M. V., Usman, M.: Exact solutions for the uniaxial extension of a mixture slab. Archives of Mechanies 40 (2), $271-282$ (1988).

[8] Gandhi, M. V., Usman, M.: The flexure problem in the context of the theory of interacting continua. SES Paper No. ESP 24.87016, presented a the 24th Annual Technical Meeting of the Society of Engng. Sci., Salt Lake City, Utah, September 1987.

[9] Treloar, L. R. G.: Swelling of a rubber cylinder in torsion: part 1. Theory. Polymer 13, 195-202 (1972).

[10] Green, A. E., Naghdi, P. M.: On basic equations for mixtures. Q. J. Mech. Appl. Math. $22(4), 427-438$ (1969).

[11] Mills, N.: Incompressible mixtures of Newtonian fluids. Int. J. Engng. Sci. 4, 97-112 (1966).

[12] Treloar, L. R. G.: The physics of rubber elasticity, 3rd ed. London: Oxford University Press 1975.

[13] Loke, K. M., Dickinson, M., Treloar, L. R. G.: Swelling of a rubber eylinder in torsion: part 2. Experimental. Polymer 13, 203-207 (1972).

[14] Gandhi, M. V., Usman, M.: Equilibrium characterization of fluid-saturated continua and an interpretation of the saturation boundary condition assumption for solid-fluid mixtures. Int. J. Engng. Sci. In press, (1989).

\author{
M. V. Gandhi and M. Usman \\ Department of Mechanical Lngineering \\ Michigan State University \\ East Lansing, MI 48824 \\ U.S.A.
}

\author{
A. S. Wineman \\ Department of Mechanical Engineering \\ and Applied Mechanics \\ University of Michigan \\ Ann Arbor, MI 48109 \\ U.S.A.
}

\author{
K. R. Rajagopal \\ Department of Mechanical Engineering \\ University of Pittsburgh \\ Pittsburgh, PA 15261 \\ U.S.A.
}

